\title{
Sequential Decay Distortion of Goldhaber Model Widths for Spectator Fragments
}

\author{
Y. G. Ma, ${ }^{1}$ 月 R. Wada,${ }^{1}$ K. Hagel, ${ }^{1}$ M. Murray, ${ }^{1}$ A. Ono, ${ }^{2}$ J. S. \\ Wang, ${ }^{1, \dagger}$ L. J. Qin, ${ }^{1}$ A. Makeev, ${ }^{1}$ P. Smith, ${ }^{1}$ and J. B. Natowitz ${ }^{1}$ \\ ${ }^{1}$ Cyclotron Institute, Texas A\&M University, College Station, Texas 7r843-3366 \\ ${ }^{2}$ Department of Physics, Tohoku University, Sendai, 980-8578, Japan
}

(Dated: November 1, 2018)

\begin{abstract}
Momentum widths of the primary fragments and observed final fragments have been investigated within the framework of an Antisymmetrized Molecular Dynamics transport model code (AMD-V) with a sequential decay afterburner (GEMINI). It is found that the secondary evaporation effects cause the values of a reduced momentum width, $\sigma_{0}$, derived from momentum widths of the final fragments to be significantly less than those appropriate to the primary fragment but close to those observed in many experiments. Therefore, a new interpretation for experiemental momentum widths of projectile-like fragments is presented.
\end{abstract}

PACS numbers: 25.70.Pq, 25.70.Mn, 02.70.Ns

Measurement of the momentum widths of projectilelike fragments (PLF) emitted in relatively peripheral nuclear collisions have long been viewed as a means of obtaining detailed information on the intrinsic Fermi momentum distribution in the nucleus $11,2,3,4,5,6,6$, 8, 9. 10, 11, 12, 13, 14. This information is fundamental in testing theoretical models of the nucleus and, in recent years, has taken on further importance in calculations related to the production of secondary fragmentation beams now being employed to study both structure and reactions far from stability 15]. Such studies, which explore a much broader range of neutron to proton asymmetry than previously accessible, are providing new insights into nuclear structure and nuclear astrophysics and offer the possibility of probing the nature of nucleonic matter in much greater detail [16].

In this Letter, we re-examine the validity of the Goldhaber Model [1] within the framework of a nonequilibrium transport model, namely the AMD-V model of Ono et al 17] and study the effects of sequential decay on the observed momentum widths of final-state spectator fragments with the help of an evaporation code GEMINI [18]. We find that the Goldhaber Model works well in describing the momentum widths of the emerging primary fragments produced in the spectator fragmentation. We find, further, that reduced momentum widths derived from the observed momentum widths of the final fragments are narrower than the reduced widths characterizing the primary fragment momentum distributions. This reflects the influence of the light particle evaporation from the primary fragments. Applying the Goldhaber Model without taking this evaporation contribution into account leads to an erroneous interpretation regarding the primary fragmentation step.

In the pioneering work of Goldhaber [1], it was suggested that the momentum widths of PLFs are determined by the intrinsic Fermi motion of the constituent nucleons which are removed from projectile during the break-up process. Assuming that $\left(A_{0}-K\right)$ nucleons are suddenly removed from a nucleus which originally has $A_{0}$ nucleons, a nucleus consisting of $K$ nucleons will emerge. For this fragment of $K$ nucleons, Goldhaber showed that the momentum width, $\sigma$, could be expressed as :

$$
\sigma=\sigma_{0} \sqrt{\frac{K\left(A_{0}-K\right)}{A_{0}-1}}
$$

where $\sigma_{0}$, the reduced momentum width, is related to the intrinsic Fermi motion of a single nucleon. If the projectile nucleons have a mean square momentum in the projectile frame equal to $(3 / 5) P_{F}^{2}$, where $P_{F}$ is the Fermi momentum, then that a momentum dispersion with $\sigma_{0}^{2}$ $=(1 / 5) P_{F}^{2}$ is expected. Based upon electron scattering measurements of Fermi momenta [19] $\sigma_{0}$ is expected to be $\simeq 112-116 \mathrm{MeV} / \mathrm{c}$ [1] . However, experimental results

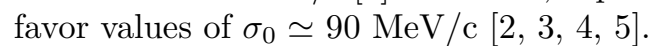

In an attempt to explain this difference, Bertsch [6] treated the correlations between the momenta of individual nucleons localized in space and calculated a corrected reduced width for ${ }^{40} \mathrm{Ar}$ fragmentation which was $17 \%$ smaller than Goldhaber's prediction. Murphy [7] considered the fact that the fragment is also a Fermi gas, ie., that there also exists a phase space constraint on the nucleon momenta in the projectile fragments which was not taken into account in the Goldhaber Model [1], and predicted fragment momentum distributions which are narrower than those observed. In a purely kinematical semi-classical model Gan et al. [8] showed that the momentum width of a fragment suddenly broken off from a Fermi distribution is sensitive to the single-particle distribution but the quantitative difference with the observed values still remains. Recently, Besliu et al. [5] took into account the dependence of $\sigma_{0}$ on the apparent temperature of the PLFs, which are excited during the collision. In this case, the projectile fragmentation process is assumed to be a slower process and the width governed by the Fermi distribution of nucleon momenta in the excited projectile as discussed by Bauer [9]. In contrast to 
most results for longitudinal momentum widths, Brady et al. observed broader transverse momentum widths than those predicted by the Goldhaber Model [10, 11, These were explained by a collective motion: a bounceoff which imparts an additional transverse momentum to the spectators and results in wider momentum widths, especially for heavier projectiles.

In recent years, several attempts have been made to provide a systematic phenomenological description of the available data [4, 12, 13] and the basic theoretical models have been extended [5]. In most of the theoretical work on the momentum widths of PLFs, analytical techniques have been employed to explore the problem. One paper, that of Gossiaux et al. [14], has employed a QMD model calculation to explore the properties of spectator matter in $600 \mathrm{MeV} /$ nucleon collisions of $\mathrm{Au}$ with $\mathrm{Au}$ and concluded that the underlying physics is more complicated than that generally assumed in analytical approaches.

The initial stage of the reaction studied in this paper, $200 \mathrm{MeV} / \mathrm{u}{ }^{40} \mathrm{Ar}+{ }^{27} \mathrm{Al}$, has been simulated using the AMD-V model 20]. AMD models have been very successfully used to study the static nature of light nuclei [21]. In this model, calculation of the minimum energy states can be carried out to define initial ground states of the colliding nuclei.

In the AMD-V model the stochastic branching process of the wave packet diffusion is incorporated. The widths of the wave packets in each stochastic branch are kept constant and the dynamics of the widths of the wave packets calculated by the Vlasov equation is incorporated as a stochastic diffusion process of the centroids of the wave packets. This model was successfully applied to multifragmentation events in the ${ }^{40} \mathrm{Ca}+{ }^{40} \mathrm{Ca}$ reaction at $35 \mathrm{MeV} / \mathrm{u}$ [22] and ${ }^{64} \mathrm{Zn}+{ }^{58} \mathrm{Ni}$ reactions [23] at $35-$ $79 \mathrm{MeV} / \mathrm{u}$. In the present calculations, the Gogny force [24], which gave the best fit in these previous analyses, was used as an effective interaction. This Gogny force gives a momentum-dependent mean field and an incompressibility of $228 \mathrm{MeV}$ for infinite nuclear matter. We note further that in our initialization of the projectile and target ground states in the AMD-V model, we get an initial width of the nucleon momentum distribution of $\sim 105 \mathrm{MeV} / \mathrm{c}$ for ${ }^{40} \mathrm{Ar}$ projectile and $\sim 101 \mathrm{MeV} / \mathrm{c}$ for the ${ }^{27} \mathrm{Al}$ target. These values are slightly less than expected from electron scattering measurements of Fermi momenta, ie. $112-116 \mathrm{MeV} / \mathrm{c}$ [19], but are still in the reasonable range.

The calculation was started at $\mathrm{t}=0$, with a distance of $15 \mathrm{fm}$ between the centers of the projectile and target in the beam direction. Each event was followed up to $t$ $=300 \mathrm{fm} / \mathrm{c}$. However, considering that we are initially interested in the properties of primary fragments, we analyze the AMD-V results at an earlier time, when the two dominant spectators just separate from each other and before significant evaporation can occur.

Calculations were carried out in the peripheral collision zone, ie. $\mathrm{b}=6-8$ fm. 10000 events are calculated. Primary fragment masses and their excitation energies were extracted at $t_{s}=60 \mathrm{fm} / \mathrm{c}$. Fragments were identified using a configuration space coalescence technique with a coalescence radius of $5 \mathrm{fm}$, but the size of the fragments depends only slightly on the coalescence radius.

The primary fragments which are isolated in this way are excited. In order to simulate experimental data as closely as possible, a modified version of the GEMINI statistical model code [18 has been used as an afterburner to follow the de-excitation of these excited fragments. In this modified version, discrete levels of the excited states of light fragments with $Z \leq 14$ are taken into account and the Hauser-Feshbach formalism is extended to treat the particle decay from a parent nucleus up to $50 \mathrm{MeV}$ excitation energy. In the calculation, care was taken to follow the entire de-excitation cascade so that the distribution of initial parent nuclei (primary fragments from AMD-V) leading to each observed final fragment could be derived and the contributions of nucleon evaporation and mass loss to the momentum widths of final fragments could be determined.

In order to study the primary fragments of spectator fragmentation, we first defined, in each event, the heaviest fragment with a positive parallel velocity in the center of mass as a projectile-like fragment and the heaviest fragment with a negative parallel velocity in the center of mass as a target-like fragment (TLF). We then analyzed the three components of the momenta of the PLF and TLF fragments. Since the the collisions are peripheral, the primary PLF and TLF can be cleanly separated.

These primary fragment momentum distributions can be described by a Gaussian shape characterized by a width $\sigma$. The primary fragment mass numbers and the three momentum widths, $\sigma_{P x}, \sigma_{P y}$ and $\sigma_{P z}(Z$ is in the beam direction and $X$ is in the direction of the impact parameter axis) are plotted in Fig. 11 as a function of the PLF and TLF fragment masses at $b=6-8 \mathrm{fm}$. The widths of the primary fragments are denoted by open circles and the dashed lines represent the results of fits to the Goldhaber Model expression (Eq.(1)). In order to obtain better fits, we allow both the initial mass $A_{0}$ and the reduced width, $\sigma_{0}$ to be free parameters. The fit parameters are shown in upper-right corner. The initial masses are close to the original masses of projectile or target and the average of the reduced widths of the three momentum components is $\sim 105 \mathrm{MeV} / \mathrm{c}$ for PLFs and $110 \mathrm{MeV} / \mathrm{c}$ for TLFs. The values of $\sigma_{0}$ are very close to the initial nucleon Fermi momentum widths of the AMD-V ground states as mentioned before. Consequently, it appears that the Goldhaber model works well for the primary fragments.

However, the calculation indicates that, the primary fragments are excited: the mean value of excitation energy is about $1.7 \mathrm{MeV} / \mathrm{u}$ and $2.7 \mathrm{MeV} / \mathrm{u}$ for PLFs and TLFs, respectively. To evaluate the effect of secondary 

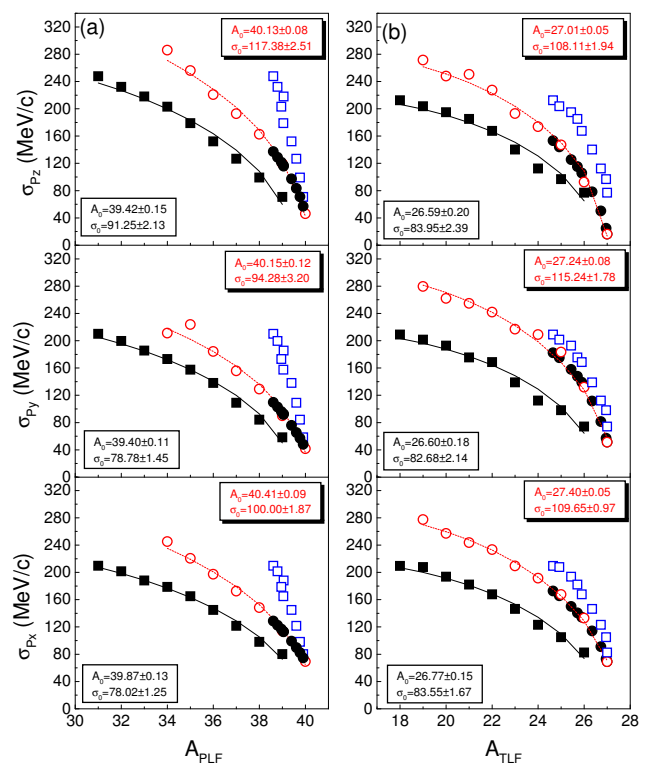

FIG. 1: The three momentum widths $\sigma_{P z}$ (upper), $\sigma_{P y}$ (middle) and $\sigma_{P x}$ (lower) as a function of the mass of PLF (left) and TLF (right) fragments. The open circles depict the momentum widths of the AMD-V primary fragments as a function of the mass of primary fragments and the dashed lines indicate the Goldhaber Model fits. The solid squares show the momentum widths of final fragments as a function of the mass of final fragment and the solid lines represent Goldhaber Model fits to those results. The open squares represent the momentum widths of final fragments as a function of the reconstructed average mass of the primary fragment. The solid circles depict the reconstructed momentum widths as a function of the reconstructed average mass of the primary fragment. See details in text.

particle emission on the masses and momentum widths we used the AMD-V results at $t_{s}$ as input to the GEMINI calculation. As a result of the subsequent statistical deexcitation the final mass distribution becomes broader but the PLF and TLF components can still be identified and the momentum widths for PLF and TLF final fragments can be determined.

The solid squares in Fig. 1 represent the momentum widths of the final fragments, observed after the secondary de-excitation. The results have also been fit with the Goldhaber Model expression (the solid line) and the extracted apparent primary mass $A_{0}$ and width $\sigma_{0}$ is shown on lower-left corner. The mass parameters remain close to the initial masses of the projectile and target but the $\sigma_{0}$ values become significantly smaller, manifesting mean values $\sim 83 \mathrm{MeV} / \mathrm{c}$. Obviously, these values approach to the typical values of $90 \mathrm{MeV} / \mathrm{c}$ observed experimentally (recall that the AMD-V ground state has a slightly lower Fermi momentum than those from exper-

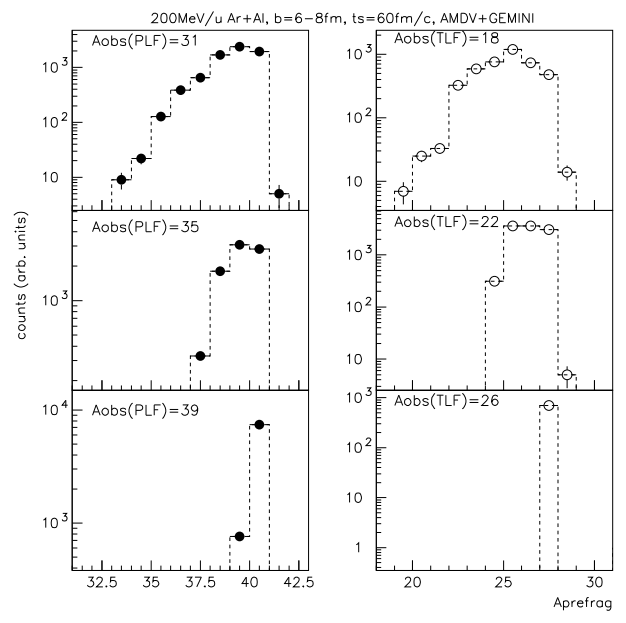

FIG. 2: The primary fragment mass distributions for some selected $\mathrm{PLF}$ and TLF final fragments for $200 \mathrm{MeV} / \mathrm{u}{ }^{40} \mathrm{Ar}+{ }^{27} \mathrm{Al}$ at $6-8$ fm.

iments). It appears that the sequential decay plays an important role in causing the reduced momentum width of the final fragments to be narrower than that of the intrinsic nucleon momentum width. This leads us a relatively simple explanation for the experimentally reported momentum widths of PLF fragments.

Why does the sequential decay make the reduced width decrease and not increase? In order to answer this question, we have tracked the secondary decay paths and obtained the distribution of parent nuclei (primary fragments) which lead to each of the observed final fragments. Fig. 2 shows some samples of the primary fragment distributions which contribute to some selected final fragments. Clearly, the farther removed the final fragment from the primary parent nuclei, the broader the distribution of primary fragments which contribute to that final fragment.

If we plot the observed momentum width for the final fragments as a function of their average primary fragment masses, as represented by the open squares in Fig. 1, we immediately see that there is a large increase of momentum width in comparison with the original primary fragment width, denoted by open circles. It is, of course, natural that the sequential decay will make the momentum distribution wider.

Knowing the parentage of a final fragment we can reconstruct the primary width distribution leading to that fragment. The reconstructed width for the average parent fragments can be written as: $\sigma_{\text {recons }} \equiv$ $\sqrt{\sum_{i} p_{i} \sigma_{\text {Prim }}(i)^{2}}$, where $p_{i}$ is the fractional contribution of primary fragments to the final observed fragments. The results are indicated by the solid circles in Fig. 1 . As required by this procedure, the results fall on the curves defining the primary fragment momentum widths. The difference in widths represented by these points and those represented by the open squares reflect evaporation 


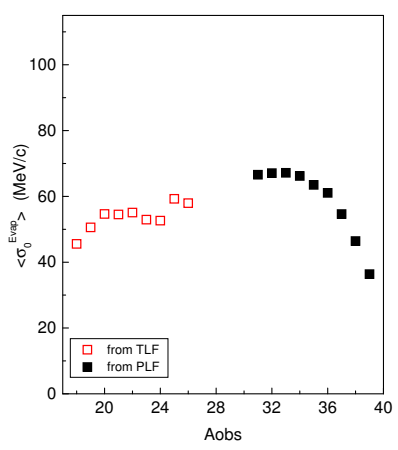

FIG. 3: Mean reduced momentum widths for light particle emission from PLF and TLF primary fragments for $200 \mathrm{MeV} / \mathrm{u}{ }^{40} \mathrm{Ar}$ $+{ }^{27} \mathrm{Al}$ at $6-8 \mathrm{fm}$.

effects.

If we pursue the analogy to the Goldhaber Model, then from the decay calculations we can also define $\sigma_{0}^{E v a p}$, a reduced width. This width characterizes the evaporation step and can readily be compared with the momentum width which results from the intrinsic nucleon momentum distribution from the primary step. We define the relationship between the width of the momentum distribution of final fragments of mass $A_{o b s}$ and those of the primary fragments $A_{\text {Prim }}(i)$ which de-excite to produce $A_{\text {obs }}$ :

$$
\left.\sigma_{\text {Aobs }}^{2}=\Sigma_{i} p_{i}\left[\sigma_{\text {Prim }}^{2}+\left(A_{\text {Prim }}(i)-A_{o b s}\right)\left(\sigma_{0}^{\text {Evap }}\right)^{2}\right)\right]
$$

where $p_{i}$ is the fractional contribution of $A_{\text {Prim }}(i)$ to the final observed fragments $\left(A_{o b s}\right)$ and $\sigma_{0}^{\text {Evap }}$ is the contribution of particle evaporation to the momentum width. Fig. 3 presents the mean value of $\sigma_{0}^{\text {Evap }}$ over Px, Py and $\mathrm{Pz}$ directions as a function of the final fragment mass. We see that $\sigma_{0}^{E v a p}$ is generally in the range of $30-70 \mathrm{MeV} / \mathrm{c}$, significantly lower than $P_{F} / \sqrt{5}$ which characterize the primary break-up.

For comparison, we also checked ${ }^{40} \mathrm{Ar}+{ }^{27} \mathrm{Al}$ at $\mathrm{b}=$ 4 - $6 \mathrm{fm}$, the similar values and the same conclusion was drawn. In addition, we also checked our technique to extract $\sigma_{0}$ with the fixed $A_{0}$ as is typically done in experimental analysis of initial projectile and target masses in the fit procedure with Goldhaber Model rather than the free parameter of $A_{0}$. The conclusions are not changed.

In summary, the momentum widths of the primary fragments and final observed fragments produced in the reaction $200 \mathrm{MeV} /$ nucleon ${ }^{40} \mathrm{Ar}+{ }^{27} \mathrm{Al}$ were investigated using an AMD-V calculation with a statistical model afterburner. It is found that the Goldhaber Model works well for the primary fragments formed in spectator fragmentation. The momentum widths of the primary fragments are basically related to the initial Fermi momenta in the projectile or target. However, since the primary fragments are excited, they are de-excited by light particle evaporation. This secondary decay decreases the observed masses and increases the observed momentum widths of the primary fragments. As a consquence, it makes the reduced width parameter $\sigma_{0}$, derived from a Goldhaber Model fit to the data, narrower by almost 20 $\mathrm{MeV} / \mathrm{c}$ than the initial Fermi momentum width, consistent with many experimental observations. Given that evaporation components can be experimentally determined from particle- fragment correlation measurements, even in very complex reactions [25], this result suggests that observed width distributions could be corrected for secondary decay and the Fermi momentum distribution of the primary fragment could be probed in relatively low intensity radioactive beam experiments. This would allow the extension of such measurements over a much larger range in $\mathrm{N} / \mathrm{Z}$ ratio and provide even more stringent tests of our microscopic models of asymmetric nuclear matter.

This work was supported by the the U.S. Department of Energy and the Robert A. Welch Foundation.

* On leave from Shanghai Institute of Nuclear Research, Chinese Academy of Sciences, Shanghai 201800, CHINA

$\dagger$ On leave from Institute of Modern Physics, Chinese Academy of Sciences, Lanzhou 730000, CHINA

[1] A. S. Goldhaber, Phys. Lett. B 53, 306 (1974).

[2] Y. P. Viyogi et al., Phys. Rev. Lett. 42, 33 (1974).

[3] D. E. Greiner et al., Phys. Rev. Lett. 35, 152 (1975).

[4] J. Reinhold et al., Phys. Rev. C 58, 247 (1998).

[5] C. Beşliu et al., Phys. Rev. C 60, 024609 (1999).

[6] G. Bertsch, Phys. Rev. Lett. 46, 472 (1981).

[7] M. Murphy, Phys. Lett. B 135, 25 (1984).

[8] H. H. Gan et al., Phys. Lett. B 234, 4 (1990).

[9] W. Bauer, Phys. Rev. C 51, 803 (1995).

[10] F. P. Brady et al., Phys. Rev. Lett. 60, 1699 (1988); W. B. Christie et al., Phys. Rev. C 48, 2973 (1993); F. P. Brady et al., Phys. Rev. C 50, R525 (1994); J. L. Chance et al., Phys. Rev. C 64, 014610 (2001).

[11] J. Dreute et al., Phys. Rev. C 44, 1057 (1991); C 47, 415 (1993).

[12] D. J. Morrissey, Phys. Rev. C 39, 460 (1989).

[13] A. Schüttauf et al., Nucl. Phys. A 607, 457 (1996).

[14] P. B. Gossiaux et al., Nucl. Phys. A 619, 379 (1997).

[15] H. Geissel et al., Ann. Rev. Nucl. Part. Sci. 45, 163 (1995).

[16] See, for example, "RIA Physics White Paper". R. Casten and W. Nazarewicz, (2000). http://www.nscl.msu.edu/ conferences/riaws00/

[17] A. Ono et al., Prog. Theo. Phys. 87, 1185 (1992).

[18] R. J. Charity et al., Nucl. Phys. A 483, 371 (1988).

[19] E. J. Moniz et al., Phys. Rev. Lett. 26, 445 (1971).

[20] A. Ono and H. Horiuchi, Phys. Rev. C 53, 845 (1996).

[21] H. Horiuchi et al., Z. Phys. A 349, 279 (1994); Nucl. Phys. A 588, 474 (1993).

[22] R. Wada et al., Phys. Lett. B 422, 2 (1998).

[23] R. Wada et al., Phys. Rev. C 62, 034601 (2000). 
[24] J. Dechargé and D. Gogny, Phys. Rev. C 21, 1568 (1980).

[25] N. Marie et al., Phys. Rev. C58, 256 (1998). 